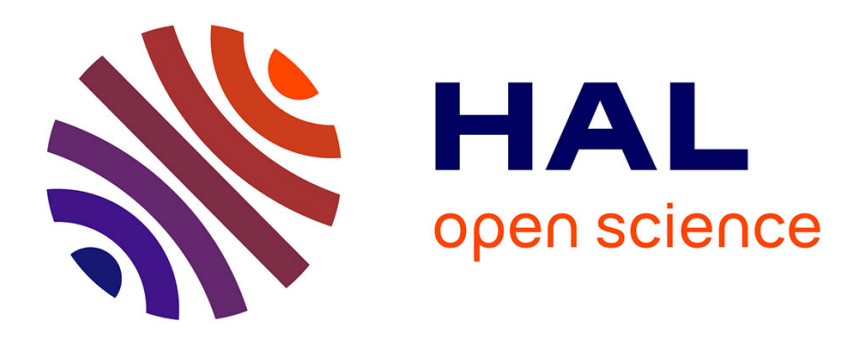

\title{
On a three-dimensional implementation of the baker's transformation
}

Philippe Carrière

\section{To cite this version:}

Philippe Carrière. On a three-dimensional implementation of the baker's transformation. Physics of Fluids, 2007, 19, pp.118110. 10.1063/1.2804959 . hal-00272157

\section{HAL Id: hal-00272157 \\ https://hal.science/hal-00272157}

Submitted on 15 Jun 2012

HAL is a multi-disciplinary open access archive for the deposit and dissemination of scientific research documents, whether they are published or not. The documents may come from teaching and research institutions in France or abroad, or from public or private research centers.
L'archive ouverte pluridisciplinaire HAL, est destinée au dépôt et à la diffusion de documents scientifiques de niveau recherche, publiés ou non, émanant des établissements d'enseignement et de recherche français ou étrangers, des laboratoires publics ou privés. 


\title{
On a three-dimensional implementation of the baker's transformation
}

\author{
Philippe Carrière \\ Laboratoire de Mécanique des Fluides et d'Acoustique, UMR CNRS 5509-Université de Lyon, \\ Ecole Centrale de Lyon-Université Lyon 1-INSA Lyon, 69134 Ecully cedex, France
}

(Received 10 August 2007; accepted 9 October 2007; published online 27 November 2007)

\begin{abstract}
A three-dimensional, steady flow configuration intended to mimic the baker's map is studied by means of numerical simulation. The Poincaré sections computed from a finite element solution of the velocity field show that the behavior is dominated by chaotic advection. The value obtained for the Lyapunov exponent is very close to the theoretical value of $\ln 2$ predicted by the baker's map. (C) 2007 American Institute of Physics. [DOI: 10.1063/1.2804959]
\end{abstract}

The baker's map is probably one of the most famous mathematical transformations in the theory of conservative dynamical systems. It is a classical reference example in books and introductory articles on the subject. ${ }^{1-3}$ Recall that the baker's map is obtained by iterating the elementary transformation $S$ defined on the unit square by

$$
S(x, y)=\left\{\begin{array}{l}
\left(2 x, \frac{1}{2} y\right) \bmod 1, \quad \text { if } 0 \leqslant x<\frac{1}{2}, \\
\left(2 x, \frac{1}{2}(y+1)\right) \bmod 1, \quad \text { if } \frac{1}{2} \leqslant x<1 .
\end{array}\right.
$$

Heuristically, the specific behavior of the map appears when considering how the upper and lower half of the square are mapped when the transformation $S$ is repeatedly applied. As sketched in Fig. 1, successively squeezing in the $y$ direction while stretching in the $x$ direction, cutting in two and then stacking the right half above the left, causes what is initially a two-strip domain to become four strips after one iteration. Repeating the iteration $n$ times produces successively $8,16,32, \ldots, 2^{n}$ alternating strips of depth $1 / 2^{n}$. The baker's map is a mixing transformation since, in the limit $n \rightarrow \infty$, the "unit square becomes completely filled with an infinite number of alternating red and yellow lines" 3 and thus any infinitesimal region of the square contains the same proportion of red and yellow.

As emphasized by Wiggins and Ottino, ${ }^{3}$ the baker's map is not only a didactic introductory example, it also possesses very interesting properties; in particular, the facts that the stretching and contraction directions are constant and uniform over the domain and that cutting and stacking is thought to be better than stretching and folding. ${ }^{3}$ Trying to reproduce exactly this transformation by some fluid flows requires the introduction of a spatially discontinuous flow field whose physical relevance is doubtful. Nevertheless, some practical mixing systems have been proposed in the literature which make use of the separation/stacking mechanism, the key underlying concept in the baker's map. A way to implement this mechanism is to consider appropriately designed three-dimensional (3-D) flows. For instance, the well-known Kenics mixer is thought to approach such behavior and several other systems have also been proposed, especially in the context of microfluidics. ${ }^{4-8}$ However, threedimensionality adds a degree of complexity to the analysis and the exact flow field inside these mixers is not known, making analytical approaches a hard task. In addition, experimental investigations are rather complicated, especially for micromixers, ${ }^{7,8}$ as are numerical simulations, ${ }^{9-11}$ so that, while some quantitative results are available, they neither allow a clear characterization of chaotic mixing nor comparisons between the theoretical model (the baker's map) and the practical system (the real mixer). In particular, being able to determine the presence and size of nonchaotic regions is essential. Here, a three-dimensional stationary continuous mixer configuration, which is intended to approach closely the transformation $S^{n}$, is considered. Its working principle is sketched in the bottom part of Fig. 1 so as to allow a comparison with the baker's map; a view of a three-element configuration is shown in Fig. 2. A basic element of the system is composed of a converging " $\mathrm{T}$ " followed by a diverging " $T$ " at right angles, the two outlets of the diverging " $T$ " are then connected to the converging " $\mathrm{T}$ " of the next element via two opposite ducts. The converging " $\mathrm{T}$ " plays the role of the "stretching/squeezing" step while the diverging one plays the role of the "cutting" step. The outer ducts, appropriately designed so that red and yellow regions are inverted in each of them, ensure the "stacking" step. If red and yellow colored fluid entering the first element forms a "two-strip" domain in the first "T," it should form a "four-strip" domain in the converging "T" of the second element, just as in the baker's transformation. The same reasoning applied to the second element leads to "eight strips" at the entrance of the third element, and so on. The system of Fig. 1 has clear similarities with the one proposed by Gray et al. ${ }^{7}$ However, in the present work, a symmetric design is used so as to avoid any additional side effects when comparing with the mathematical transformation $S^{n}$. Indeed, the real fluid flow which take place in a such a system does not follow exactly the scheme of principle and this already influences (greatly?) the mixing properties, a point which requires a refined study.

In this paper, the emphasis is put on the academic viewpoint, so that the study is restricted to creeping flows (Stokes approximation), a fairly usual approach in chaotic advection. In addition, the geometry is designed to make numerical simulation as simple - and accurate - as possible, but realistic. Nevertheless, it must be noted that practical realization of the system is possible. A macroscale system would not be much more complicated to manufacture than the twisted 

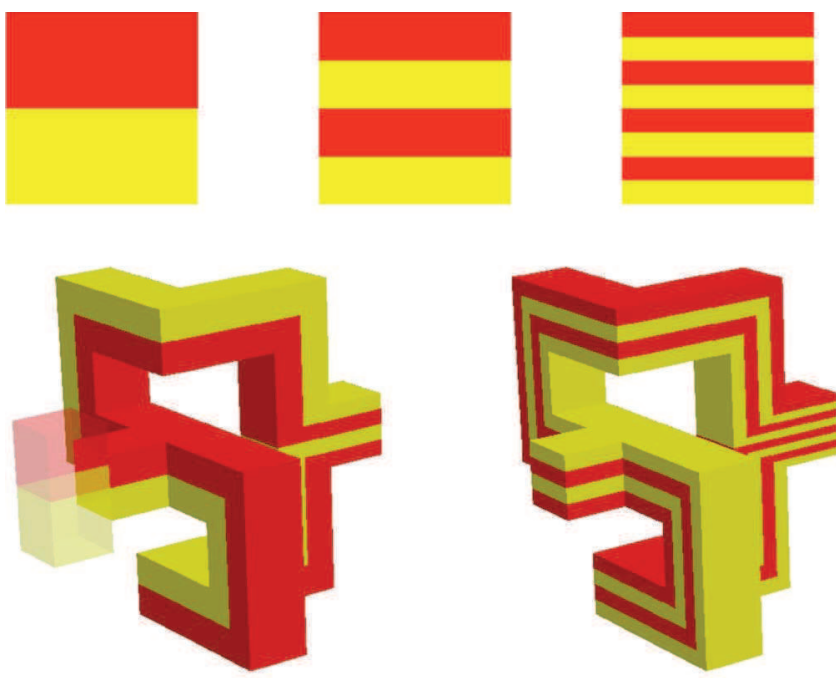

FIG. 1. (Color online) Top: baker's transformation of the unit square after two iterations. Bottom: sketch of mixing action of the present flow after two elements.

pipes mixer $^{12}$ (see Ref. 13 for a realistic prototype). Even downsizing to microfluidic devices, while less straightforward, is not intractable by means of either multiple superposed layers (for polydimethylsiloxane systems) or double sided (for glass systems) manufacturing; such techniques have been successfully applied in Refs. 7 and 8. Regarding the state-of-the-art in microfluidics, ${ }^{14}$ still there is a need for investigating such micromixer concepts even if interesting, different ones have already been proposed in the literature ${ }^{15-19}$ there is also a need for clearer characterizations of such mixers. A salient feature of the present type of system is that the mixing process is induced by the primary flow, while many other ones ${ }^{13,15,16}$ are based on a secondary flow action, which can have limited amplitude. This system has the potential to produce larger stretching rates for the same energy cost. Another feature of this system is that, as a channel or pipe flow, it offers a large surface-to-volume ratio and is therefore expected to be a very efficient heat exchanger (as is the twisted pipe mixer).

Studying the system of Fig. 2 beyond a qualitative analysis requires knowledge of the corresponding velocity field. In the following, we consider a pressure driven flow with the no-slip condition along the walls. Since the system is composed of a succession of identical elements, the velocity field may be assumed spatially periodic when neglecting inlet and outlet effects. Therefore, the determination of the flow field

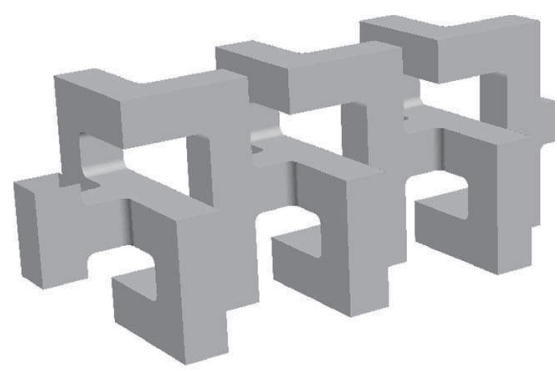

FIG. 2. The present mixing system in a three basic elements configuration.

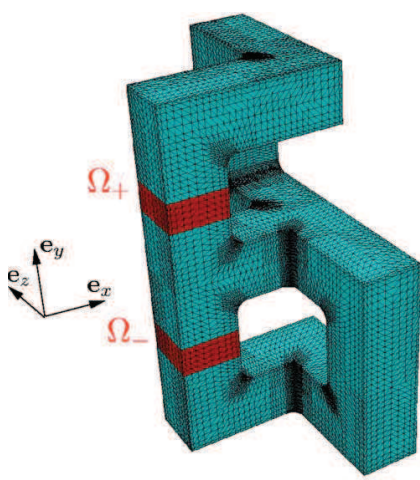

FIG. 3. (Color online) The computational mesh corresponding to the "closed" configuration used to simulate the flow field in a basic element of the system of Fig. 2. In red (dark gray), the location of the subdomains where a constant, volume force is imposed.

may be restricted to a single element with the velocity field satisfying periodic conditions between the inlet and the outlet and the pressure field being also periodic up to an additive constant (the mean pressure difference between the inlet and the outlet). Due to computational considerations, a quite equivalent problem involving a closed rather than opened geometry was solved instead. For this, as is clear from the plot of the mesh in Fig. 3, the design of the outer ducts has been modified in a symmetric manner so that they are now connected to the inlet ports of the same element.

In this closed configuration, the driving pressure of the open system has been replaced by a volume force $\mathbf{f}$, which is constant inside each of the plane-parallel subdomains $\Omega_{-}$and $\Omega_{+}$[marked in red (dark gray) in Fig. 3] located on both sides of the inlet of the double "T." Since imposing a constant volume force in part of a straight pipe leads to the same velocity field as in the purely pressure driven case, here it is expected that the generated flow does not depart much from its counterpart in the open system. In the Stokes approximation, with $\mathbf{u}$ and $p$ the nondimensional velocity and pressure, respectively, the momentum equation reduces to

$$
-\nabla^{2} \mathbf{u}=-\nabla p+\mathbf{f}
$$

with $\mathbf{f}$ given by $\mathbf{f}(\mathbf{x})= \pm f_{ \pm} \mathbf{e}_{y}$ for $\mathbf{x}$ in $\Omega_{ \pm}$and 0 elsewhere, where $f_{-}$and $f_{+}$are two constants to be specified. Note that, since we are interested in the characterization of the "line mixer" properties, any time dependence have been neglected in Eq. (2). All quantities are assumed to be nondimensionalized using the width $w$ of the pipe as the length scale and $\mu F^{-1} w^{-1}$ as the time scale, with $\mu$ the dynamic viscosity of the fluid and $F$ the characteristic magnitude of the volume forcing. Numerical solutions of Eq. (2), together with the continuity constraint and no-slip boundary conditions, have been obtained using a finite element method similar to the one used in Ref. 20, but with quadratic approximation for $\mathbf{u}$. Fluid particle trajectories and their associated Lyapunov exponents are computed as presented in Ref. 21.

As usual, the Lagrangian properties of the flow are characterized by means of Poincaré sections and Lyapunov exponents. For this purpose, the section plane at $x=1.5$, corresponding to the middle of the "double-T," is considered. Two typical choices of parameters, i.e., $f_{-}$and $f_{+}$, have been con- 

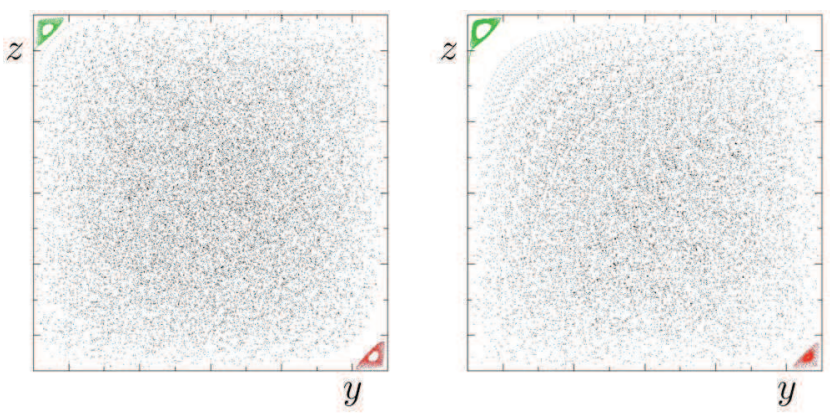

FIG. 4. (Color online) Poincaré sections at $x=1.5$. On the left, $f_{+}=f_{-}$; on the right, $f_{+}=3 f_{-}$.

sidered: $f_{+}=f_{-}$corresponds to the desired induced motion where the flow rates inside each outer duct are the same; $f_{+}=3 f_{-}$, has been adopted to investigate the sensitivity of the mixing process to the presence of some kind of imperfection inducing different flow rates inside a real system. Figure 4 reveals that, as expected, chaotic advection is effectively generated by the flow. The behavior of fluid particle trajectories is dominated mainly by the central chaotic region, covering most of the section even in the "unfavorable" case: $f_{+}=3 f_{-}$. The key result is obtained when determining the Lyapunov exponent associated with the chaotic region in the two cases. Their convergence with the number of iterations of the Poincaré map is shown in Fig. 5, where we also show that the Lyapunov exponent associated with nonchaotic regions converges to a reasonably small value. To facilitate the comparison, the straight line corresponding to the value $\ln 2$ (the value of the Lyapunov exponent of the baker's map) has been plotted. Clearly, the value of the Lyapunov exponent obtained in the favorable case $f_{+}=f_{-}$(about 0.68 ) is very close to $\ln 2$, so that the present system clearly succeeds in reproducing, at least in the mean, the same global stretching rate as expected from the baker's transformation. In the unfavorable case, the Lyapunov exponent (about 0.45) clearly departs from $\ln 2$. Thus, while the essential qualitative features of the fluid motion are similar in the two cases, after only three elements a typical material line is only half as

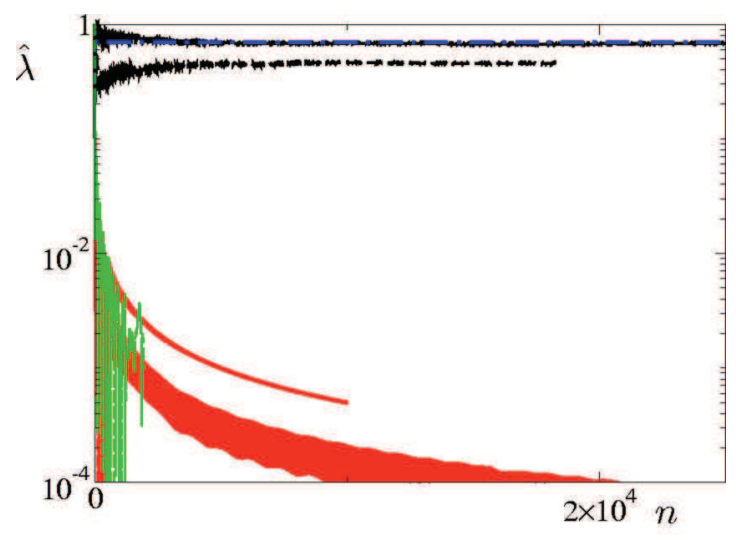

FIG. 5. (Color online) Convergence of the maximum Lyapunov exponent $\hat{\lambda}$ with the number of iterations $(n)$ of the spatial mapping for solid line: $f_{+}$ $=f_{-}$(left plot of Fig. 4); dashed line: $f_{+}=3 f_{-}$(right plot of Fig. 4). Horizontal dot-dashed line is for $\hat{\lambda}=\ln 2$. long in the unfavorable case as in the favorable case. This point must be kept in mind for applications where symmetries of the flow are not easy to preserve.

As far as stretching rate is concerned, for most fluid particles and asymptotically in time, the effect of the present system is thus very similar to the one of the baker's map $S^{n}$. However, there are two regions, located at opposite corners, which exhibit nonchaotic behavior. [The reader may have noted that the nonchaotic regions in fact exhibit slowly converging/diverging orbits, a signature of numerical approximation of the velocity field (whose divergence depends on the mesh size), a behavior we did not observed in previous works (Refs. 18 and 21). Here, investigations with different meshes indicated that it is probably related to the presence of re-entrant corners (especially in the "double-T," but also all along the duct) where the pressure problem is illconditioned accounting to our formulation.] Even if they are rather small, the presence of such nonchaotic regions is not satisfactory since they are well known to be barriers to mixing. ${ }^{22-24}$ We numerically checked that increasing the distance between the two "T" junctions does not significantly change the presence of these islands. Now, noting that the location of these islands follows from the symmetry of the system suggests that it would probably be better for successive elements to be inverted (one time the upward duct on the left, one time on the right, etc.). However, this requires a different computational domain than the one used here (since the spatial period is now two elements), so this investigation is postponed to future work. In addition, the appearance of islands may very well be related to the square cross section used in the present system and studying different crosssection shapes would be useful.

Another issue of interest is that, from the mechanical engineering viewpoint, characterizing the chaotic behavior by means of $\hat{\lambda}$ is insufficiently informative since no characteristic time scale is involved. Rather, one has to return back to the time Lyapunov exponent $\lambda$ which, following Ref. 25, is related to $\hat{\lambda}$ by $\hat{\lambda}=\lambda \bar{\tau}$, with $\bar{\tau}$ the mean time between two successive Poincaré section points. A first estimate of a characteristic mixing time can then be obtained, but it remains necessary to account for the energy consumption of the system. For a stationary flow, the power supply $\mathcal{P}$ due to the forcing term is dissipated by the action of viscous forces, implying the dot product of the rate of deformation tensor $\boldsymbol{\sigma}$ by itself. Now, $\boldsymbol{\sigma}$ is also the origin of stretching, thus introducing the nondimensional number

$$
\mathcal{N}_{\lambda}=\lambda\left(\frac{1}{V_{\Omega}} \int_{\Omega} \boldsymbol{\sigma}: \boldsymbol{\sigma} \mathrm{d} \Omega\right)^{-1 / 2}=\lambda\left(\frac{\mathcal{P}}{2 \mu V_{\Omega}}\right)^{-1 / 2},
$$

with $V_{\Omega}$ the volume of $\Omega$, gives a measure of the mixing system's "efficiency." Here, we find $\mathcal{N}_{\lambda}=0.01636$ when $f_{+}$ $=f_{-}$, and $\mathcal{N}_{\lambda}=0.009817$ when $f_{+}=3 f_{-}$. As a basis for comparison, for the "academic" 3-D flow we studied in Refs. 22 and 23, we found $\mathcal{N}_{\lambda}=0.0711$ (for the most efficient flow); this latter finding is a significantly better result but the freeslip boundary condition for the academic flow probably favors a minimal energy loss. However, this suggests a basis for a comparison exercise from an optimization viewpoint 
concerning existing devices both of the present kind and of different underlying concepts.

The author would like to gratefully acknowledge Benoît Pier for his help and suggestions, and one of the anonymous referees for his proofreading of the manuscript.

${ }^{1}$ M. A. Lichtenberg and A. J. Lieberman, Regular and Chaotic Dynamics (Springer, New York, 1992.

${ }^{2}$ J. M. Ottino, The Kinematics of Mixing: Stretching, Chaos and Transport (Cambridge University Press, New York, 1989).

${ }^{3}$ S. Wiggins and J. M. Ottino, "Foundations of chaotic mixing," Philos. Trans. R. Soc. London, Ser. A 362, 937 (2004).

${ }^{4}$ H. Mensinger, T. Richter, V. Hessel, J. Döpper, and W. Ehrfeld, "Microreactor with integrated static mixer and analysis system," in Micro Total Analysis Systems, edited by A. van den Berg and P. Bergveld (Kluwer, Dordrecht, 1994), pp. 237-243.

${ }^{5}$ N. Schwesinger, T. Frank, and H. Wurmus, “A modular microfluid system with an integrated micromixer," J. Micromech. Microeng. 6, 99 (1996).

${ }^{6}$ J. Branebjerg, P. Gravesen, J. P. Krog, and C. R. Nielsen, "Fast mixing by lamination," in Proceedings of the 9th IEEE Workshop on Micro Electro Mechanical Systems (IEEE, New York, 1996), pp. 441-446.

${ }^{7}$ B. L. Gray, D. Jaeggi, N. J. Mourlas, B. P. van Drieënhuizen, K. R. Williams, N. I. Maluf, and G. T. A. Kovacs, "Novel interconnection technologies for integrated microfluidic systems," Sens. Actuators, A 77, 57 (1999).

${ }^{8}$ H. Chen and J.-S. Meiners, "Topologic mixing on a microfluidic chip," Appl. Phys. Lett. 84, 2193 (2004).

${ }^{9}$ O. Byrde and M. L. Sawley, "Optimization of a Kenics static mixer for non-creeping flow conditions,” Chem. Eng. J. 72, 163 (1999).

${ }^{10}$ M. Regner, K. Östergren, and C. Trägårdh, "Effects of geometry and flow rate on secondary flow and the mixing process in static mixers-a numerical study," Chem. Eng. Sci. 61, 6133 (2006).

${ }^{11}$ J. M. MacInnes, A. Vikhansky, and R. W. K. Allen, "Numerical characterisation of folding flow microchannel mixers," Chem. Eng. Sci. 62, 2718 (2007).
${ }^{12}$ S. W. Jones, O. M. Thomas, and H. Aref, "Chaotic advection by laminar flow in a twisted pipe," J. Fluid Mech. 209, 335 (1989).

${ }^{13}$ C. Castelain, D. Berger, P. Legentilhomme, A. Mokrani, and H. Peerhossaini, "Experimental and numerical characterisation of mixing in a steady spatially chaotic flow by means of residence time distribution measurements," Int. J. Heat Mass Transfer 43, 3687 (2000).

${ }^{14}$ H. A. Stone, A. D. Stroock, and A. Ajdari, "Engineering flows in small devices, microfluidics toward lab-on-a-chip," Annu. Rev. Fluid Mech. 36, 381 (2004)

${ }^{15}$ A. D. Stroock, S. K. W. Dertinger, A. Ajdari, I. Mezic, H. A. Stone, and G. M. Whitesides, "Chaotic mixer for microchannels," Science 295, 647 (2002).

${ }^{16}$ A. Dodge, A. Hountondji, M. C. Jullien, and P. Tabeling, "Spatiotemporal resonances in a microfluidic system," Phys. Rev. E 72, 056312, (2005).

${ }^{17}$ F. Raynal, F. Plaza, A. Beuf, Ph. Carrière, E. Souteyrand, J.-R. Martin, J.-P. Cloarec, and M. Cabrera, "Study of a chaotic mixing system for DNA chip hybridization chambers," Phys. Fluids 16, L63 (2004).

${ }^{18}$ F. Raynal, A. Beuf, F. Plaza, J. Scott, Ph. Carrière, M. Cabrera, J.-P. Cloarec, and E. Souteyrand, "Towards better DNA chip hybridization using chaotic advection," Phys. Fluids 19, 017112 (2007).

${ }^{19}$ M. K. McQuain, K. Seale, J. Peek, T. S. Fisher, S. Levy, M. A. Stremler, and F. Haselton, "Chaotic mixer improves microarray hybridization," Anal. Biochem. 325, 215 (2004).

${ }^{20} \mathrm{Ph}$. Carrière and $\mathrm{D}$. Jeandel, "A $3 \mathrm{D}$ finite element method for the simulation of thermoconvective flows and its performances on a vector-parallel computer,” Int. J. Numer. Methods Fluids 12, 929 (1991).

${ }^{21} \mathrm{Ph}$. Carrière, "Lyapunov spectrum determination from the FEM simulation of a chaotic advecting flow," Int. J. Numer. Methods Fluids 50, 555 (2006).

${ }^{22}$ V. Toussaint, $\mathrm{Ph}$. Carrière, and F. Raynal, "A numerical Eulerian approach to mixing by chaotic advection," Phys. Fluids 7, 2587 (1995).

${ }^{23} \mathrm{~V}$. Toussaint and $\mathrm{Ph}$. Carrière, "Diffusive cut-off of fractal surfaces in chaotic mixing," Int. J. Bifurcation Chaos Appl. Sci. Eng. 9, 443 (1999).

${ }^{24}$ X. Z. Tang and A. H. Boozer, "A Lagrangian analysis of advectiondiffusion equation for a three dimensional chaotic flow," Phys. Fluids 11, 1418 (1999).

${ }^{25}$ J. P. Eckmann and D. Ruelle, "Ergodic theory of chaos," Rev. Mod. Phys. 57, 617 (1985) 